\title{
Pedagogical Feasibility of Using Systems on the Web-interface for Implementating the Interdisciplinary Nature of Training
}

\author{
Irena Robert ${ }^{1, a *}$ \\ 1 Institute of Educational Management of the Russian Academy of Education, 105062, 16
} Zhukovskogo str., Moscow, Russia

arena_robert@mail.ru

${ }^{*}$ Corresponding author

Keywords: intellectualization of information interaction, subjects, educational process, intellectualization of the learning process, information interaction, information and communication technologies, convergence of pedagogical science and ICT, convergent pedagogical technologies, scientific and pedagogical practices

\begin{abstract}
The article describes the capabilities of systems operating on the web-interface, presents the conditions for the intellectualization of information interaction between the subjects of the educational process for the implementation of the interdisciplinary nature of training related to the concept of convergence of pedagogical science and information and communication technologies.
\end{abstract}

\section{Introduction}

The current stage in the development of informatization of education $([4,6])$ is associated with the use of information systems that operate on the basis of a web-interface, which are actively introduced into the educational process and initiate information interaction, both between subjects of the educational process and between them and interactive information resources. The use of information systems operating on a web-based interface ensures the development of open (online) education, expands the range of copyright developments in the field of creating an electronic educational resource, and intensifies the automation of monitoring learning outcomes.

\section{Methodology}

The identified capabilities of systems operating on a web-interface, determine the pedagogical expediency of their use. On this basis, theoretical and practical approaches to ensuring the intellectualization of information interaction between the subjects of the educational process in the context of ensuring the interdisciplinary nature of learning are identified.

\section{Research}

Analysis of systems operating on the web-interface allowed to identify and describe their capabilities:

- "Embedding" in lectures or practical classes of information contained in databases in dynamic and informative fragments (webinar, social networking materials), including recording them, processing them, and transmitting them to remote users;

- Organization in a synchronous and (or) asynchronous mode of information interaction between the subjects of the educational process from any computer platform;

- Ensuring the processing of any information, user registration, his identification, control of the results of his learning with testing, with statistical processing of learning results while ensuring a remote access to information resources;

- The Wikipedia-like organization of the development of an information resource or educational content and its use in conditions of free access for each member of the community;

- Examination of jointly developed content in accordance with pedagogical and ergonomic requirements for educational products being implemented on the basis of ICT [4]. 
The implementation of the capabilities of information systems operating on a web-interface determines the pedagogical expediency of using them to ensure the intellectualization of information interaction between the subjects of the educational process:

- Provision of an audiovisual contact with a student with the objects of the studied subject area while ensuring dialogue by the system in the presence of feedback;

- Imitation, modeling of an educational situation or a studied object, a process in conditions of managing the studied objects, processes, collecting, processing, transmitting and using information;

- Provision of freedom to search for audiovisual information, including "libraries of educational and methodological experience", "libraries of methodical decisions", "libraries of imitations of the objects or processes under study";

- Consideration of educational information in various aspects of its implementation, from different points of view, on the basis of various conceptual approaches, in various modes of educational activity, on the basis of which a student makes his assumptions, creates hypotheses, draws conclusions;

- Providing the following: a tool for the study of abstract images and concepts; a tool for modeling, imitation of the studied objects, phenomena, both real and virtual; a tool for designing objects or processes of the objective world in accordance with the conceptual and methodical approaches;

- Providing a non-contact control of various virtual objects or processes displayed on the screen;

- The implementation of interaction with objects or participation in processes that are displayed on the screen, as well as the impact on their changes and development;

- Designing on the screen of certain constructions of models of the studied and (or) studied objects, processes (both real and virtual) adequately with their mental abstract interpretation in their visualization and dynamic transformations.

Based on the above, let us turn to the issue of realizing the capabilities of systems operating on a web interface to ensure the interdisciplinary nature of training related to the concept of convergence of pedagogical science and information and communication technologies. At the same time, the convergence of pedagogical science and information and communication technologies is considered as a coincidence, similarity, mutual transfer of the characteristic properties of pedagogical science and ICT, as the coincidence of information technology methods with the methods inherent in pedagogical science, and, as a result, their mutual influence on each other, their evolutionary convergence [1], [6]. In addition, the convergence of pedagogical science and ICT is considered as the mutual penetration of information technology methods into the methods of pedagogical science and, as a result, their evolutionary convergence and even their merging. The convergence of pedagogical science and ICT initiates the development of the interdisciplinary nature of education due to the mutual influence on each other of various areas of pedagogical science and ICT. At the same time, the identification and implementation of the conditions of mutual influence and penetration of the characteristic features of ICT and pedagogical technologies, as well as the identification of their similarities in functions and structures, serve as the basis of the interdisciplinary nature of training and the formation of combined subject areas, which are studied using the ICT tools.

In connection with the idea of convergence of pedagogical science and ICT, let us present the substantive grounds for describing the conditions for the implementation of the interdisciplinary nature of education.

1. Combining (partial or fragmentary) the content of various subject areas or the mutual penetration of the content of individual disciplines is pedagogically expedient for overcoming narrowly professional and narrowly objective interests. This ensures:

- Formation of the world perception as a whole on the basis of interdisciplinary educational programs; 
- Formation of interdisciplinary approaches to education for the organization of joint educational productive activities in solving generalized problems.

2. The coincidence and similarity of the characteristic features of pedagogical science and information and communication technologies (ICT) are based on the following:

- Presence of special formalisms for the presentation of declarative and procedural knowledge in an electronic form coincides with the characteristic features of pedagogical science (formalization and structuring of the content of educational material in the form of formalized structures);

- Automation of the collection, search, selection of essential information, its processing, replication, storage, transmission coincide with the characteristic features of pedagogical science in terms of automating the implementation of various types of information activities for the collection, selection, processing, replication, production, storage, and transmission of educational information.

3. The mutual transfer of characteristic features of pedagogical science and ICT is based on the following:

- Production and formalization of information are transferred to the characteristic features of pedagogical science (formalized presentation (visually, graphically, or textually) of logically completed blocks of information adequately to the content of educational materials);

- Algorithmization of information is transferred to a characteristic feature of pedagogical science (learning algorithmization, representing algorithmic instructions for solving problems of a certain class);

- Informational interaction between users and an interactive information resource are transferred to a characteristic feature of pedagogical science (the creation of an informational and educational environment, which are the conditions of informational and educational interaction between the subjects of the educational process);

- Automation of information interaction between the objects presented on the screen, automating user interaction with ICT tools while eliminating the need for regulatory support are transferred to a characteristic feature of pedagogical science (automation of information interaction between subjects of the educational process and an interactive information resource).

4. The coincidence of ICT methods with the methods of pedagogical science is based on the following:

- The algorithmization method, the method of selecting solutions for solving problems, the design method coincide with the methods of pedagogical science (the method of algorithmic learning, the trial and error method in solving problems of a particular class, the project method);

- The information modeling method; methods of logical-linguistic modeling for solving problems of non-formalizable areas of knowledge and fields of activity coincide with the methods of pedagogical science (methods of creating information models of the objects or processes under study or models of qualimetric assessment of students' preparedness level).

Thus, pedagogical technologies and ICT are converging [5] in the following positions:

- By methods (algorithmization method, modeling method, classification method, formalization method, trial and error method, method of producing and using information);

- By means of automation (visual presentation of the results; search, selection, control of the results of educational activities; information interaction/conversation);

- By means of formalization, production and presentation of information;

- By forms of organization of activity (informational activity on information retrieval by essential features; on collecting, processing, replicating, transmitting, producing information; on formalizing information).

Next, we turn to the possible negative consequences of the influence on the personality of active and systematic information interaction. Modern researchers ([2,3]) focuse attention on the possible negative 
consequences associated with the intervention in the individual's inner world of unnaturally vivid impressions of virtual objects and subjects. The enthusiasm of having an individual with vivid impressions, which often lead him away from the problems of real life, is quite obvious. At the same time, there is often a relationship between people in their real life and, which is more dangerous, a distortion in the mind of a person of the reality around him. At the same time, it is necessary to identify and describe the following: laws of "virtualization" of information interaction in the conditions of using information systems operating on the web interface between students or between a student and an interactive source of information resource; psychological characteristics of students' perception of audiovisual information presented by means of information and communication technologies; psychological features of replacing real communication with "virtual communication" in the implementation of information interaction both between students and between them and an interactive source of information; conditions of psychological support or rehabilitation of a student engaged in "virtual communication".

Consideration of the philosophical aspects of the information interaction of the student with the objects of the virtual screen world convinces of the need to study the influence on the user of the "deceptive nature" of objects of the virtual world and communication with them. In addition, at present, the philosophical aspects of the identification (self-identification) of an individual in the virtual world have not been identified or described; philosophical, spiritual, structural, and substantial characteristics of a modern person, whose vital activity is carried out in social networks, have not defined.

A number of authors (such as [2], [3], [4]) clearly demonstrate that the interaction of a student with virtual on-screen objects, his participation in on-screen virtual computer games, in chat rooms, in Internet communities, etc., lead to the effect of "losing the reality." In addition, the user's self-presentation in the "virtual world" is often carried out anonymously, not adequately with reality, since each user can present himself on the screen in different interpretations, with any emotional overtones and, at the same time, find a communication partner in the networks. It also leads to the replacement of the reality by a "virtual" one, in which permissiveness, anonymity, the ability to control virtual objects and processes leads to an imbalance in the behavior of an individual in the real world.

Considering the psychological comfort of the user's communication with virtual objects of "screen worlds", it should be noted that a student, interacting with the objects of the virtual world, finds himself in situations that require all-consuming attention and abstraction from the real world, which creates an increased emotional background of communication with screen objects and enhances user alienation from the reality. The negative is the isolation of a student from the real world, especially in terms of the audiovisual presentation of screen objects, processes, and their transformations. Thus, it is necessary to ensure the following: psychological security; comfort in the information interaction of a student with the objects of the virtual world; opportunities to log out and "return to the real world".

Of particular importance is the need to comply with the pedagogical and ergonomic requirements for the organization of educational information interaction when using pedagogical products developed using ICT. The solution of this issue implies: implementation of instructive and regulatory acts and documents approved by the relevant government bodies; performance of work with information systems; compliance with approved sanitary and hygienic norms and rules; examination of educational products developed using ICT.

\section{Conclusion}

Based on the above, the requirements for information systems operating on a web interface are defined for the implementation of the interdisciplinary nature of training:

Pedagogical requirements: systematic tutor support for students; administering educational processes (distributed educational activities) in geographically distributed groups of students; development and distribution of training materials and information systems; availability of information and educational environment corresponding to different learning styles, regardless of the territorial location of the subjects of the educational process and the distributed information educational resources.

Technological requirements: organization of educational activities using information contained in databases and used in the implementation of educational programs; providing information processing with 
the help of information technologies, technical means, as well as information and telecommunication networks; providing information transmission over communication lines; providing information interaction of students and teachers; availability of educational technologies being implemented with the use of information and telecommunication networks; indirect (at a distance) interactions of students and teachers.

\section{Acknowledgments}

The work was conducted in the 2nd quarter of 2018 as part of the State Assignment on the Program of Basic Scientific Research of the State Academies of Sciences for 2013-2020, approved by the Order of the Government of the Russian Federation of December 3, 2012 No. 2237-p, on the topic "Development of informatization of education in the context of information security of a person" (state registration № 14.07.00.20.01.04).

\section{References}

[1] Kovalchuk, M. V. (2011). The convergence of science and technology: A breakthrough in the future. Russian Nanotechnology, 6(1-2), pp.13-23.

[2] Robert, I. V. (2014). Convergence of the science of education and information technology as an evolutionary convergence of science and technology. Information Environment of Education and Science, 20, pp. 25-67.

[3] Mukhametzyanov, I. Sh. (2014). Medical and psychological bases of functioning of the information and educational space (for teachers, administration of educational institutions, and researchers). Kazan Pedagogical Journal, 1, pp. 27-43.

[4] Mukhametzyanov, I. Sh. (2012). Guidelines for the prevention of negative medical consequences of the use of ICT in education. Moscow, Russia: IIE RAS.

[5] Robert, I. V. (2014). Theory and methods of education informatization (psychological, pedagogical, and technological aspects). Moscow, Russia: BINOM. Laboratory of Knowledge.

[6] Robert, I. V. (2015). Scientific and pedagogical practices as a result of the convergence of pedagogical science and information and communication technologies. Pedagogical Informatics, 3, pp. 27-41.

[7] Robert, I.V., \& Lavina, T.A. (Eds.) (2011). Explanatory dictionary of terms of the conceptual apparatus of informatization of education. Moscow, Russia: BINOM. Laboratory of knowledge. 